\title{
Open charm production in photoproduction and DIS at HERA
}

\author{
Sergey Fourletov ${ }^{* \dagger}$ \\ University of Toronto, Canada \\ E-mail: fourl@mail.desy.de
}

Charm production has been measured at low $Q^{2}$ in the transition region between photoproduction and deep inelastic scattering with the ZEUS detector at HERA using an integrated luminosity of about $80 \mathrm{pb}^{-1}$. The kinematic region of the measurement is $p_{t}\left(D^{*}\right)>1.5 \mathrm{GeV},\left|\eta\left(D^{*}\right)\right|<1.5$, $0.05<y<0.85,0.05<Q^{2}<0.7 \mathrm{GeV}^{2}$. The decay channel $D^{*+} \rightarrow D^{0} \pi_{s}^{+}$with $D^{0} \rightarrow K^{-} \pi^{+}$ and corresponding antiparticle decay were used to identify $D^{*}$ mesons. The data are compared with the predictions of next-to-leading-order $\mathrm{QCD}$. The relative production between the $e^{+} p$ and the $e^{-} p$ data sets is also measured at HERA II using $73 \mathrm{pb}^{-1} e^{ \pm} p$ data and compared to previous results.

International Europhysics Conference on High Energy Physics

July 21st - 27th 2005

Lisboa, Portugal

\footnotetext{
* Speaker.

${ }^{\dagger}$ on behaf of the ZEUS Collaboration
} 


\section{Introduction}

Charm quarks are produced copiously in ep collisions at HERA. At sufficiently high photon virtualities, $Q^{2}$, the production of charm quarks constitutes up to $30 \%$ of the total cross section.

Previous measurements of $D^{*}$ cross sections indicate that the production of charm quarks in DIS in the range $\left(1<Q^{2}<1000 \mathrm{GeV}^{2}\right)$ is consistent with calculations in quantum chromodynamics QCD in which charm is produced through the boson-gluon-fusion (BGF) mechanism. This implies that the charm cross section is directly sensitive to the gluon density in the proton.

Also inclusive photoproduction $\left(Q^{2} \approx 0\right)$ of $D^{*}$ mesons has been measured with the ZEUS detector at HERA using an integrated luminosity of $78.7 \mathrm{pb}^{-1}$ and shows roughly consistent results with perturbative QCD predictions.

A good addition to this measurements is the transition region $\left(0.05<Q^{2}<0.7 \mathrm{GeV}^{2}\right)$ between photoproduction and DIS, which can be reached in ZEUS [2] with the Beam Pipe Calorimeter (BPC) [3] - a detector covers a small area near beam pipe, not reachable by the main Calorimeter.

\section{Charm in Photoproduction}

Charm-quark photoproduction has been extensively studied at HERA using $D^{*}$ mesons [8]. In Fig. 1 the cross sections measured by the ZEUS collaboration were compared to next-to-leadingorder (NLO) [5] and next-to-leading-logarithmic (NLL) [6] calculations. The experimental results generally lie above the expectations, in particular in the proton direction. Recently, fixed-order plus next-to-leading-logarithmic (FONLL) calculations for charm photoproduction at HERA have become available. These calculations incorporate mass effects up to NLO and the resummation of transverse-momentum logarithms up to NLL level. The FONLL predictions do not give a better description of the data than NLO. For large $p_{T}\left(D^{*}\right)$, the FONLL predictions are below the NLO calculations, considerably underestimating the data.

\section{Charm Production in transition Photoproduction $\rightarrow D I S$ region}

As charm in DIS $\left(Q^{2}>1.5 \mathrm{GeV}^{2}\right)$ is dominated by the boson-bluon-fusion process and there in a significant resolved contribution to charm photoproduction $\left(Q^{2} \approx 0\right)$, it is interesting to investigate the transition region between photoproduction and DIS.

The total cross section measured in the kinematic region $0.05<Q^{2}<0.7 \mathrm{GeV}^{2}, 0.05<y<$ $0.85, p_{T}\left(D^{*}\right)>1.5 \mathrm{GeV},\left|\eta\left(D^{*}\right)\right|<1.5$ is $\sigma=10.1 \pm 1.0 \frac{+1.1}{-0.8} \mathrm{nb}$, which is in good agreement with the the prediction from the HVQDIS program: $\sigma=8.6 \frac{+1.9}{-1.8} \mathrm{nb}$. The uncertainty on the HVQDIS calculation is about the same size as the uncertainty on the measurement.

The measured differential $D^{*}$ cross sections as a function of $Q^{2}, \mathrm{y}, p_{T}\left(D^{*}\right)$ and $\eta\left(D^{*}\right)$ for the data are shown in Fig.2 and compared with the ZEUS NLO PDF implemented in the HVQDIS program [4] . Predictions using an alternate PDF, CTEQ5F3, are also shown. The measured differential cross sections are described over the full measured kinematic region by the NLO QCD predictions from the HVQDIS program.

This analysis was also compared to previous ZEUS measurements of $D^{*}$ production in DIS [1] made in the kinematic region for $1.5<Q^{2}<1000 \mathrm{GeV}^{2}, 0.02<y<0.7,1.5<p_{T}\left(D^{*}\right)<15 \mathrm{GeV}$ 


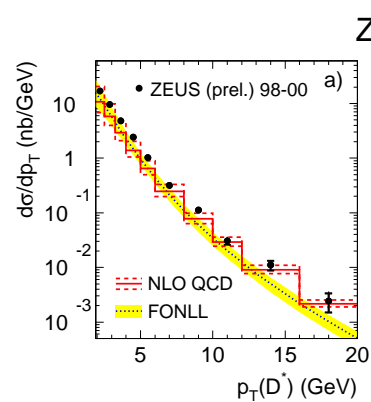

ZEUS
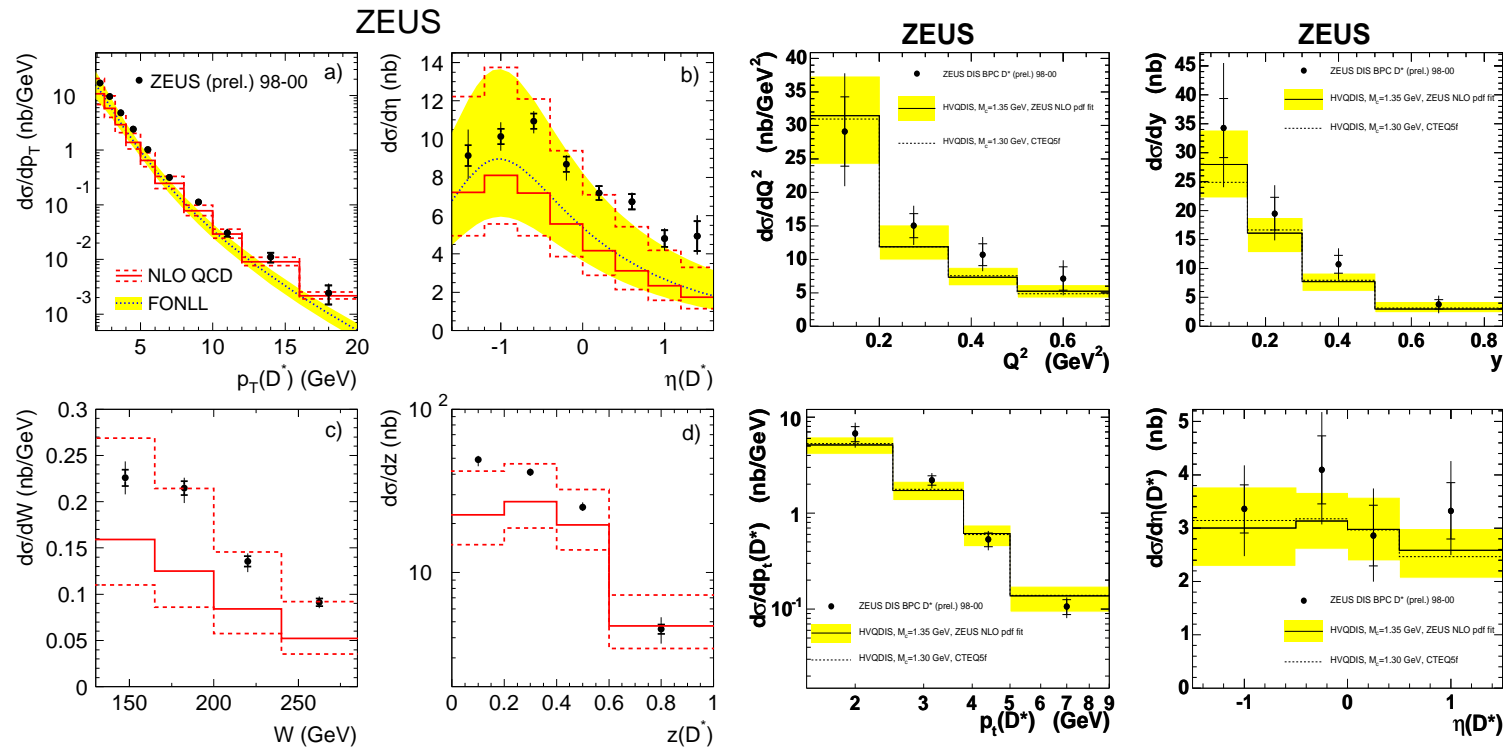

Figure 1: Inclusive $D^{*}$ photoproduction

Figure 2: BPC $D^{*}$ differential cross sections
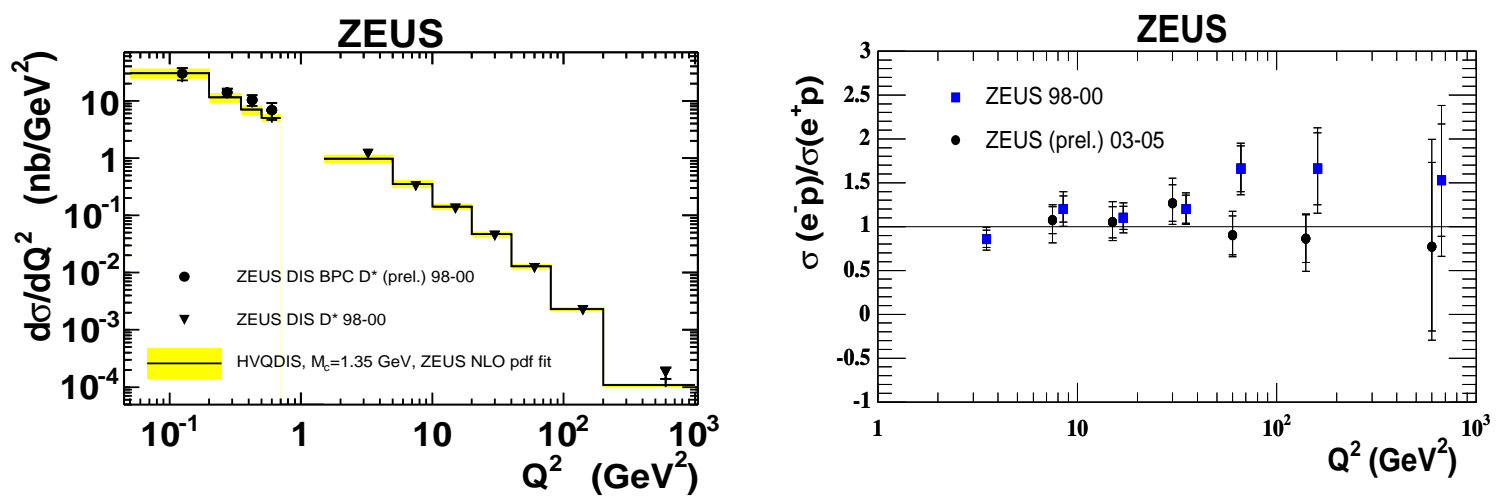

Figure 3: The $D^{*}$ production cross-section as a function of $Q^{2}$ in transition region and previous results in DIS [1]

Figure 4: The ratio $r^{e^{-} p} / r^{e^{+} p}$ for the 2004-2005 running period (solid dots) compared to the ZEUS measurement for the 1998-2000 running period.

and $\eta\left|\left(D^{*}\right)\right|<1.5$. In order to be able to directly compare the results presented here the kinematic region of this analysis was restricted to $0.02<y<0.7$ and recalculated.

On Fig.3 NLO QCD describes charm production in DIS over 4 orders of magnitude in $Q^{2}$ and over 5 orders of magnitude in the cross section.

\section{Charm in DIS}

In the most recently published ZEUS measurement [1], made using $65 \mathrm{pb}^{-1} e^{+} p$ data and $17 \mathrm{pb}^{-1} e^{-} p$ data, it was observed that the $D^{*}$ production rate, $r=N / \mathscr{L}$, for $e^{-} p$ was systematically higher than in the $e^{+}$data set, and rising with $Q^{2}$ (Fig.4). The ratio $r^{e^{-} p} / r^{e^{+} p}$ was equal to 
$1.67 \pm 0.21$ (only statistical errors are given) for $40<Q^{2}<1000 \mathrm{GeV}^{2}$. Such a difference was not expected from any physics process, and so the phenomenon was treated as a statistical fluctuation.

The measurement was repeated using HERA II data : $40 \mathrm{pb}^{-1} \mathrm{e}^{+} \mathrm{p}$ and $33 \mathrm{pb}^{-1} \mathrm{e}^{-} \mathrm{p}$ in the range $5<Q^{2}<1000 \mathrm{GeV}^{2}$ collected during 2003-5 running period [7]. The ratio $r^{e^{-} p} / r^{e^{+} p}$ is compared to the HERA I ZEUS results from 1998-2000. A virtue of this analysis is that many of the systematic effects cancel. In total $1240 \pm 64(1118 \pm 60) D^{*}$ candidates in the kinematic region $5<Q^{2}<1000 \mathrm{GeV}^{2}, 0.02<y<0.7,1.5<p_{T}\left(D^{*}\right)<15 \mathrm{GeV}$ and $\left|\eta\left(D^{*}\right)\right|<1.5$ were found in $e^{+} p\left(e^{-} p\right)$ data corresponding to a rate of $30.7 \pm 1.8 / \mathrm{pb}^{-1}\left(33.5 \pm 1.8 / \mathrm{pb}^{-1}\right)$. The rate for events with $Q^{2}>40 \mathrm{GeV}^{2}$ was $6.4 / \mathrm{pb}^{-1}\left(6.3 / \mathrm{pb}^{-1}\right)$ for $e^{+} p\left(e^{-} p\right)$ data.

The ratio $r^{e^{-} p} / r^{e^{+} p}$ in bins of $Q^{2}$ is shown in Fig.4, compared to the ZEUS HERA I values and the theoretical expectation of unity. At all values of $Q^{2}$ the value of the ratio is consistent with unity. The observation of the increase of this ratio with increasing $Q^{2}$, found previously [1], is not confirmed.

\section{Conclusion}

Charm production has been measured in DIS as a function of $Q^{2}, y, p_{T}\left(D^{*}\right)$ and $\eta\left(D^{*}\right)$ in the kinematic region $0.05<Q^{2}<0.7 \mathrm{GeV}^{2}, 0.05<y<0.85, p_{T}\left(D^{*}\right)>1.5 \mathrm{GeV},\left|\eta\left(D^{*}\right)\right|<1.5$ The measurement of charm in the transition region between DIS and photoproduction extends previous results in DIS to lower $Q^{2}$ The HVQDIS calculation produces a good description of the measured data. In particular, NLO QCD describes the dependence on $Q^{2}$ of the data over four orders of magnitude in $Q^{2}$. The combination of both measurements shows that the slope of $d \sigma / d Q^{2}$ changes with $Q^{2}$; at high $Q^{2}$ the slope is steeper than at low $Q^{2}$. This behaviour is well reproduced by the NLO QCD calculations.

A study of the ratio, $r^{e^{-} p} / r^{e^{+} p}$, of the production rate of $D^{*}$ mesons in the HERA II was made using data taken by ZEUS during the 2003-5 running period. The ratio observed to be consistent with unity at all values of $Q^{2}$, as would be expected within the Standard Model. The results support the assertion that the higher value of the ratio observed previously was due to a statistical fluctuation.

\section{References}

[1] ZEUS Coll., S. Chekanov et al.,Phys. Rev.D 69, 0120004 (2004).

[2] ZEUS Coll., M.Derrick et al., Phys.Lett. B 293 (1992) 465.

[3] B. Surrow,Ph.D. Thesis, Hamburg University, Report DESY-THESIS-1998-004, 1998.

[4] B. W. Harris and J. Smith, Charm quark and D* cross sections in DIS... Phys. Rev. D, 2806 (1998).

[5] S. Frixione et al., Nucl. Phys. B 454, 3 (1995) ; S. Frixione et al., Phys. Lett. B 348, 633 (1995).

[6] M. Cacciari, S. Frixione and P. Nason, JHEP 0103, 006 (2001).

[7] ZEUS Coll.,S. Chekanov et al., Charm production in deep inelastic scattering using HERA II data XXII Int. Symp. on Lepton-Photon Interactions, 2005, Uppsala

[8] ZEUS Coll.,S. Chekanov et al., Measurement of $D^{*}$ photoproduction at HERA ICHEP2002, Amsterdam 